\title{
ATENÇÃO FARMACÊUTICA NA FARMÁCIA COMERCIAL
}

\author{
Alexandro Moraes da Costa ${ }^{1}$ \\ Lívia Cabral Lobo ${ }^{2}$ \\ Michel Santos da Silva ${ }^{3}$ \\ Leonardo Guimarães de Andrade 4
}

RESUMO: Os conceitos de Atenção Farmacêutica foram analisados desde a sua origem, nos Estados Unidos, e as contribuições posteriores provenientes da Espanha e do esforço de sistematização pela Organização Mundial da Saúde para compreender o processo que vem ocorrendo no Brasil. Após o abandono das farmácias comunitárias, os farmacêuticos brasileiros esperam que esse novo modelo de prática seja um caminho para o resgate do seu papel social. A filosofia que orienta a Atenção Farmacêutica, o enfoque centrado no paciente, em nosso entendimento, deve suportar filosófica e conceitualmente a reconstrução da prática farmacêutica no Brasil a fim de resgatar a relação farmacêuticopaciente nas farmácias comunitárias. A atenção farmacêutica é um serviço prestado pelo farmacêutico, por meio de uma consulta com o paciente, para que ele tenha, a partir do uso correto de medicamentos, resultados positivos que melhore a sua saúde e a qualidade de vida. O farmacêutico especializado em atenção farmacêutica é o profissional adequado para prevenir, detectar e resolver problemas relacionados ao uso de medicamentos para garantir que o paciente tenha os resultados esperados com o seu tratamento. O serviço de Atenção Farmacêutica não substitui a consulta médica. Sendo assim, o farmacêutico deve orientar o paciente a buscar a assistência necessária de outros profissionais de saúde. A atuação profissional do farmacêutico em atenção farmacêutica tem crescido e sido notada como fundamental para prevenir, identificar e resolver os problemas relacionados ao uso do medicamento. $O$ farmacêutico especializado em atenção farmacêutica contribui com seus conhecimentos para assessorar o paciente sobre o uso, dose, armazenamento, frequência, administração e horários correto de medicamentos.

Palavras- chave: Atenção farmacêutica. Paciente. Farmacêutico.

ABSTRACT: The concepts of Pharmaceutical Care were analyzed since its origin, in the United States, and subsequent contributions from Spain and the effort to systematize the World Health Organization to understand the process that has been taking place in Brazil. After abandoning community pharmacies, Brazilian pharmacists hope that this new practice model will be a way to rescue their social role. The philosophy that guides

\footnotetext{
I Estudante de farmácia na Universidade Nova Iguaçu. E-mail: alexmoraesfama@gmail.com

${ }^{2}$ Universidade Iguaçu

${ }^{3}$ Universidade Iguaçu

${ }^{4}$ Universidade Iguaçu
} 
Pharmaceutical Care, the patient-centered approach, in our understanding, must philosophically and conceptually support the reconstruction of pharmaceutical practice in Brazil in order to rescue the pharmacist-patient relationship in community pharmacies. Pharmaceutical care is a service provided by the pharmacist, through a consultation with the patient, so that he has, from the correct use of medication, positive results that improve his health and quality of life. The pharmacist specializing in pharmaceutical care is the appropriate professional to prevent, detect and solve problems related to the use of medications to ensure that the patient has the expected results with their treatment. The Pharmaceutical Care service does not replace the medical appointment. Therefore, the pharmacist must guide the patient to seek the necessary assistance from other health professionals. The professional role of the pharmacist in pharmaceutical care has grown and has been seen as essential to prevent, identify and solve problems related to the use of medication. The pharmacist specializing in pharmaceutical care contributes with their knowledge to advising the patient on the correct use, dose, storage, frequency, administration and timing of medications.

Keywords: Pharmaceutical care. Patient. Pharmacist.

\section{INTRODUÇÃO}

A participação do farmacêutico na equipe multiprofissional tem sido consolidada, e a sua proximidade da comunidade reforça a implementação de projetos promoção à saúde, tendo como local de realização a própria farmácia e a aplicação de uma nova prática: a Atenção Farmacêutica.

A Atenção Farmacêutica é uma prática que tem como principal finalidade melhorar a qualidade de vida do paciente que faz ou não uso de medicamentos. Além de otimizar o tratamento farmacológico e prevenir problemas relacionados ao uso de medicamentos. A atenção farmacêutica é o componente da prática profissional onde o farmacêutico interage diretamente com o paciente para atender suas necessidades relacionadas aos medicamentos.

Segundo Cipolle e colaboradores, a atenção farmacêutica é um processo de assistência ao paciente, lógico, sistemático e global, que envolve três etapas: a) análise da situação das necessidades do paciente em relação aos medicamentos; b) elaboração de um plano de seguimento, incluindo os objetivos do tratamento farmacológico $e$ as intervenções apropriadas; e c) a avaliação do seguimento para determinar os resultados reais no paciente. 
No Brasil, no final do ano 200o, um grupo constituído por várias entidades foi formado com o objetivo de promover a atenção farmacêutica no país, considerando as características da prática profissional local. Como consequência, em 2002, foi proposto um conceito nacional para o tema. O conceito proposto considera a promoção da saúde e, dentro dela, a orientação em saúde, como componentes do conceito de atenção farmacêutica. O Consenso definiu também os componentes da prática farmacêutica necessários ao exercício da atenção farmacêutica: a) educação em saúde, b) orientação farmacêutica, c) dispensação, d) atendimento farmacêutico, e) acompanhamento/seguimento farmacoterapêutico e f) registro sistemático das atividades, mensuração e avaliação dos resultados.

Tradicionalmente, no Brasil, o farmacêutico não tem atuação destacada no acompanhamento da utilização de medicamentos, na prevenção e promoção da saúde e é pouco reconhecido como profissional de saúde tanto pela sociedade quanto pelos demais profissionais da citada área. De maneira geral, o principal serviço prestado nas farmácias e drogarias é a dispensação de medicamentos e a qualidade dessa prática pode ser considerada abaixo do padrão, uma vez que os farmacêuticos frequentemente estão ausentes da farmácia. Em vista disto, o conceito de atenção farmacêutica sugere mudanças na atuação profissional predominante.

O medicamento é importante para a melhora da saúde, entretanto sua eficácia depende, dentre outros fatores, do seu uso racional cuja orientação é realizada pelo farmacêutico. Esse profissional, por sua vez, deve realizar atividades vinculadas à promoção da saúde, tendo em vista o bem-estar do paciente. A recente prática profissional da Atenção Farmacêutica é um importante instrumento na promoção da saúde já que nela, o paciente é o principal beneficiário das ações do farmacêutico. Para efetivar essa atividade, primeiramente os profissionais farmacêuticos precisam de conhecimento ou atualização nessa nova prática além de dispor de fontes de informação independente e imparcial sobre medicamentos.

É preciso que os proprietários de farmácias comunitárias, em especial, tomem consciência da necessidade de implantá-la em seu estabelecimento, e criem um ambiente adequado para a execução desta atividade. Atualmente, a farmácia é vista como um 
comércio e não como um estabelecimento de saúde. Quando presente, na maioria das vezes, o farmacêutico acumula funções burocráticas, assim não lhe sobra tempo para atender os pacientes de forma completa que seria a prestação de não apenas uma orientação de balcão, mas a Atenção Farmacêutica como um todo, em sua plenitude.

$\mathrm{Na}$ condição de estabelecimento que integra os sistemas de saúde, a farmácia apresenta vantagens, tais como, fácil acesso a um profissional de saúde; condições adequadas para participação em campanhas sanitárias; redução de Io gastos com tratamentos, por possibilitar intervenção primária e encaminhamento à assistência médica; aumento na observância à terapêutica farmacológica prescrita, com consequente melhoria na qualidade de vida do usuário. O farmacêutico, mais do que nunca, tem um papel importante junto à construção de um novo modelo de atenção à saúde, onde ele possa estar inserido como profissional do medicamento, atuando como referência na orientação, cumprimento, acompanhamento e monitoramento da terapia farmacológica.

\section{ORIGEM E CONCEITOS DA ATENÇÃO FARMACÊUTICA}

No século $\mathrm{XX}$, o papel do farmacêutico associava-se à produção e comercialização de produtos medicinais, além disso, esse profissional apresentava grande vínculo com equipes de saúde e com o próprio paciente. No entanto, essa atuação tradicional sofreu uma diminuição, a partir da Segunda Guerra Mundial, em função do desenvolvimento da indústria farmacêutica.

Esse fato levou a um descompasso entre a formação do profissional e as ações demandadas pela sociedade, gerando uma frustração em alguns profissionais, pois os conhecimentos adquiridos na graduação já não eram mais aplicados de forma permanente na prática diária e acabavam se perdendo. A partir de então, o farmacêutico relacionado à área assistencialista distanciou-se das equipes de saúde e dos pacientes, passando a ser visto apenas como um dispensador de produtos fabricados. Nesse contexto surgiram, na década de 1960, líderes profissionais e educadores norte-americanos que organizaram um movimento profissional com a finalidade de questionar a formação e as atitudes do farmacêutico, bem como corrigir possíveis erros cometidos no exercício de sua profissão.

O conceito clássico dessa prática, formulado por Hepler; Strand, baseia-se na 
responsabilidade farmacoterapêutica orientada com o objetivo de melhorar a qualidade de vida dos pacientes, atingindo, assim a promoção da saúde através da cura de uma doença; eliminação ou redução dos sintomas do paciente; interrupção ou retardamento do processo patológico, ou prevenção de uma enfermidade ou de um sintoma.

\section{FARMÁCIA COMUNITÁRIA}

Estabelecimento de prestação de serviços farmacêuticos de interesse público e/ou privado, articulada ao Sistema Único de Saúde, destinada a prestar assistência farmacêutica e orientação sanitária individual ou coletiva, onde se processe a manipulação e/ou dispensação de produtos e correlatos com finalidade profilática, curativa, paliativa, estética ou para fins de diagnósticos.

\section{I ACOMPANHAMENTO FARMACOTERAPÊUTICO}

É um componente da Atenção Farmacêutica e configura um processo no qual o farmacêutico se responsabiliza pelas necessidades do usuário relacionadas ao medicamento, por meio da detecção, prevenção e resolução de Problemas Relacionados aos Medicamentos (PRM), de forma sistemática, contínua e documentada, com o objetivo de alcançar resultados definidos, buscando a melhoria da qualidade de vida do usuário.

\subsection{INTERVENÇÃO FARMACÊUTICA}

É um ato planejado, documentado e realizado junto ao usuário e profissionais de saúde, que visa resolver ou prevenir problemas que interferem ou podem interferir na farmacoterapia, sendo parte integrante do processo de acompanhamento/seguimento farmacoterapêutico.

\subsection{ATENDIMENTO FARMACÊUTICO}

É o ato em que o farmacêutico, fundamentado em sua práxis, interage e responde às demandas dos usuários do sistema de saúde, buscando a resolução de problemas de saúde, que envolvam ou não o uso de medicamentos. Este processo pode compreender 
escuta ativa, identificação de necessidades, análise da situação, tomada de decisões, definição de condutas, documentação e avaliação, entre outros.

\subsection{USO RACIONAL DE MEDICAMENTOS}

É o processo que compreende a prescrição apropriada; a disponibilidade oportuna e a preços acessíveis; a dispensação em condições adequadas; e o consumo nas doses indicadas, nos intervalos definidos e no período indicado de medicamentos eficazes, seguros e de qualidade. Devido aos problemas encontrados nos serviços de saúde, falta de condições, o uso irracional de medicamentos poderá provocar danos à saúde da população e causar dependência, sendo que o atendimento médico ainda continua sendo uma forma de avaliar o paciente antes do diagnóstico e prescrição.

\section{DISPENSAÇÃO}

É o ato profissional farmacêutico de proporcionar um ou mais medicamentos a um paciente, geralmente como resposta à apresentação de uma receita elaborada por um profissional autorizado. Nesse ato, o farmacêutico informa e orienta o paciente sobre o uso adequado do medicamento. São elementos importantes da orientação, entre outros, a ênfase no cumprimento da dosagem, a influência dos alimentos, a interação com outros medicamentos, o reconhecimento de reações adversas potenciais $e$ as condições de conservação dos produtos.

\section{I ATIVIDADES DESENVOLVIDAS PELO FARMACÊUTICO NO SERVIÇO DE ATENÇÃO FARMACÊUTICA}

São atividades relacionadas com o medicamento, destinadas a apoiar as ações de saúde demandadas por uma comunidade. Envolve o abastecimento de medicamentos em todas e cada uma de suas etapas constitutivas, a conservação e controle de qualidade, a segurança e a eficácia terapêutica dos medicamentos, o acompanhamento e a avaliação da utilização, a obtenção difusão de informação sobre medicamentos e a educação permanente dos profissionais de saúde, do paciente e da comunidade para assegurar o uso racional de medicamentos. 
- Acompanhamento e avaliação da eficácia do tratamento farmacoterapêutico prescrito pelo médico;

- Aferição de pressão, temperatura corporal e glicemia,

- Administração de medicamentos;

- Orientação sobre o uso correto do medicamento;

- Intervenção no tratamento, quando necessário;

Benefícios da atenção farmacêutica para o paciente

- Facilidade de acesso e comunicação com um profissional de saúde;

- Orientação para o uso correto do medicamento;

- Esclarecimento de dúvidas;

- Acompanhamento contínuo da terapia medicamentosa;

- Verificação da efetividade do tratamento;

- Identificação e redução dos problemas relacionados ao uso do medicamento;

- Prevenção da doença ou sintomas;

- Intervenção para solução de problemas;

- Eliminação ou diminuição dos sintomas;

- Melhora na saúde;

- Melhora da qualidade de vida;

I. HABILIDADES NECESSÁRIAS PARA O FARMACÊUTICO ATUAR EM ATENÇÃO FARMACÊUTICA

- Especializar em atenção farmacêutica e ter um preparo técnico para o atendimento diferenciado e exclusivo com o paciente.

- Ter conhecimento aprofundado sobre: farmácia clínica, farmacologia, terapia não medicamentosa, interpretação de exames laboratoriais, dispensação ativa, prescrição farmacêutica, farmacoterapia e outras noções que contribuem para o uso racional de medicamentos e executar o serviço com qualidade;

- Ser simpático;

- Ter empatia; 
- Buscar sempre mais conhecimento;

- Ser comunicativo;

- Ser proativo.

\section{2 ATIVIDADES PERTINENTES À ATENÇÃO FARMACÊUTICA}

Segundo o Código de Ética da Profissão Farmacêutica, “o farmacêutico é um profissional da saúde, cumprindo-lhe executar todas as atividades inerentes ao âmbito profissional farmacêutico de modo a contribuir para a salvaguarda da saúde pública e, ainda, todas as ações de educação dirigidas à comunidade na promoção da saúde”. Sendo o farmacêutico um profissional muito próximo das pessoas e das comunidades, tem grandes possibilidades de interação e de influenciar em vários âmbitos. Dessa forma é importante que o farmacêutico desenvolva atividades que modifiquem positivamente o comportamento da comunidade, já que nesta posição estratégica, ele é capaz de orientar o paciente e atuar como agente sanitário de acesso fácil para a população e suas funções não se limitam à dispensação, devendo abranger um ato profissional muito mais amplo, em que deve expor todo seu conhecimento especializado, orientando também mudanças em hábitos alimentares e estilo de vida.

Mas isso só é possível se o profissional estiver disposto e disponível para atuar veementemente na construção da Atenção Farmacêutica, principal atividade vinculada a estes cuidados. Além desta, existem algumas atividades essenciais para considerá-la implantada.

Dentre as principais atividades que a caracterizam, inclui-se a detecção de suspeita de reações adversas e de intoxicações por medicamentos, orientação ao paciente, comunicação ao prescritor e notificação do evento. Segundo Vidotti, Silva, a implantação de ações de Atenção Farmacêutica é muito importante para aumentar a aderência ao tratamento, prevenir intoxicações, promover o uso e armazenamento de forma segura, 16 prevenir o surgimento de resultados negativos associados à medicação, melhora na qualidade de comunicação com o paciente, elaboração de educação em saúde e campanhas vinculadas às necessidades da comunidade. 
Em um programa de Atenção Farmacêutica, o farmacêutico passa a conhecer melhor o paciente. Sabe não apenas que medicamentos ele toma e de que maneira o faz, mas também como se sente com o tratamento e com seu problema de saúde. $\mathrm{Na}$ prática, o farmacêutico, de maneira organizada, coleta e avalia informações sobre o paciente, incluindo a identificação de possíveis RNMs. Identificado o problema, busca a solução, fórmula e coloca em prática um plano para corrigi-lo. Para executar este programa de atenção farmacêutica, o profissional necessita ampliar suas habilidades e conhecimentos além daqueles utilizados na prática tradicional.

Outras atividades também são muito úteis e poderiam ser desempenhadas na farmácia comunitária, respeitando a legislação sanitária: aconselhamento ao paciente; educação e promoção da saúde; aconselhamento em planejamento familiar e teste de gravidez; participação em campanhas de sanitárias, apoio à interrupção do fumo, entre outras. Através da Atenção Farmacêutica, o profissional pode levar o paciente a realizar atividades de autocuidado, a partir do aconselhamento sobre o fornecimento de um medicamento ou outro tratamento que levam os pacientes à automedicação gerar a consciência de que o paciente guarda para si o controle e a responsabilidade sobre sua saúde.

Adicionalmente, o desenvolvimento de programas de Atenção Farmacêutica deve ser feito com planejamento, implementação e avaliação dos resultados. Por isso, o registro das atividades faz parte do processo da Atenção Farmacêutica, o que permitirá a análise do acompanhamento farmacoterapêutico, se as atividades executadas melhoram o estado de saúde do paciente.

\subsection{COMO IMPLANTAR O SERVIÇO DE ATENÇÃO FARMACÊUTICA EM UMA FARMÁCIA OU DROGARIA?}

- I $\mathrm{I}^{\mathrm{a}}$ Fase: Planejamento

Conhecimento:

Antes de implantar o serviço, o farmacêutico responsável precisa adquirir conhecimento e se especializar em atenção farmacêutica. Ele deve estar plenamente capacitado para executar o serviço e atender o paciente da melhor forma possível. 
Infraestrutura:

O serviço de atenção farmacêutica requer um espaço exclusivo e diferenciado para receber o paciente. $O$ atendimento inicial pode até ser feito no balcão, mas não pode continuar nele. É preciso determinar e preparar uma sala para esse atendimento. $\mathrm{O}$ paciente precisa estar confortável no local, seguro que ninguém vai ver ou escutar o que está sendo conversado e sem interrupções. O ideal é que seja uma sala climatizada, com mesa, cadeira, computador, livros para consulta e outros itens que promovam o bem-estar, segurança e privacidade do paciente.

- $2^{\text {a }}$ Fase: Execução do serviço

A segunda fase é colocar em prática o que você planejou na primeira e seguir o POP. De forma geral, o atendimento se resume em:

- Atendimento ao paciente na sala reservada;

- Entrevista para coleta de informações sobre o paciente (patologias, medicamentos que utiliza, dosagem, frequência e como o paciente está se sentindo);

- Aferição de pressão, temperatura corporal e glicemia;

- Estudo do caso do paciente, avaliação do tratamento, intervenção quando necessário.

- Orientação ao paciente sobre o uso correto do medicamento;

- Registro e documentação do atendimento;

- Termo de Consentimento do atendimento assinado pelo paciente;

- Deixar claro ao paciente que o serviço não substitui a consulta médica.

Declaração de Serviço Farmacêutico

Após a prestação do serviço de atenção farmacêutica, o profissional deve entregar ao paciente a Declaração de Serviço Farmacêutico. Esse documento precisa conter as seguintes informações:

- Dados de identificação da instituição de saúde;

- Identificação pessoal do paciente ou do seu responsável legal;

- Medicamento prescrito e nome e inscrição no conselho profissional do médico que prescreveu o fármaco; 
- Indicação de Medicamento Isento de Prescrição (MIP) e a posologia, quando houver;

- Valores dos parâmetros fisiológicos e bioquímico, quando houver, seguidos dos valores considerados normais para o paciente;

- Frase de alerta, quando houver medição de parâmetros fisiológicos e bioquímico: “ESTE PROCEDIMENTO NÃO TEM FINALIDADE DE DIAGNÓSTICO E NÃO SUbSTitui A CONSUlta MÉdiCA OU A REALIZAÇÃO DE EXAMES LABORATORIAIS";

- Dados do medicamento administrado (nome comercial, exceto para genéricos, denominação comum brasileira, concentração e forma farmacêutica, via de administração, número do lote e número de registro na Anvisa)

- Orientação e intervenção farmacêutica;

- Data, assinatura e carimbo com inscrição no Conselho Regional de Farmácia (CRF) do farmacêutico responsável pelo serviço.

Para prestar o serviço de atenção farmacêutica você precisa planejar o que vai oferecer aos clientes e instituir normas e procedimentos de como será esse serviço. Para isso é necessário criar o Procedimento Operacional Padrão (POP). Obrigatoriamente, conforme a RDC n 44/2009, a drogaria ou a farmácia devem instituir o POP com todos os processos e normas que devem ser seguidas para a realização do serviço.

Neste POP deve conter:

- Metodologia e processos para a prestação de serviço;

- Metodologia e processos para a documentação do atendimento;

- Metodologia de avaliação dos resultados;

\subsection{ATENÇÃO FARMACÊUTICA NA FARMÁCIA COMERCIAL}

Atenção Farmacêutica é o conjunto de ações voltadas à promoção, proteção e recuperação da saúde, tanto individual como coletiva, tendo o medicamento como insumo essencial e visando o acesso de medicamentos e insumos, bem como a sua seleção, programação, aquisição, distribuição, dispensação, garantia da qualidade dos produtos e 
serviços, acompanhamento e avaliação de sua utilização, na perspectiva da obtenção de resultados concretos e da melhoria da qualidade de vida da população

\section{CONCLUSÃO}

Os dados apresentados nesse estudo mostraram que a Atenção Farmacêutica, é de fundamental importância para uma eficácia clínica no seu tratamento, pois o farmacêutico pode diminuir o uso irracional de medicamento, evitando possíveis interações medicamentosas, inclusive com alimentos, além de proporcionar informações sobre a posologia e forma de armazenamento desses medicamentos. Entretanto essa realidade ainda está distante de se tornar uma rotina em nossa vida, pois são inúmeros os fatores que contribuem para esta complexidade assistencial do farmacêutico. Dentre eles, pode-se citar a falta de apoio dos proprietários nas farmácias, dificuldades relacionadas ao ambiente de trabalho, como concorrência com os balconistas, atividades administrativas desempenhadas pelos farmacêuticos, falta de interesse pelos pacientes, a falta de contato com equipes multiprofissionais de saúde e até mesmo falta de preparação do farmacêutico para desempenhar a função. Desta forma é preciso que haja uma mudança curricular e investimento na infraestrutura da farmácia para permitir a atividade plena dessa prática.

\section{BIBLIOGRAFIA}

Bonfim, Guilherme. A Importância Do Profissional De Farmácia $\mathrm{Na}$ Atenção Farmacêutica. IIf. Revista Científica Multidisciplinar O Saber. Sp. V. ol, P. ol-02, Dezembro, 2020. Disponível Em: Https://Revistartigo/Index.Php/Rcmos/Article/View/3/7

Firmo, Wellyson. Paredes, Alcyone. Cunha, Carlos. Torres, Ayslan. Buccini, Danieli. Análise Das Prescrições Médicas De Psicotrópicos De Uma Farmácia Comercial No Município De Bacabal, Maranhão. gf. 2013. Disponível Em: Https://Www.Jmphc.Com.Br/Jmphc/Article/View/I6I/ı64

Possamai, Fabricio. Dacoreggio, Marlete. Habilidade De Comunicação Com O Paciente No Processo De Atenção Farmacêutica. 2of. 2017. Universidade Do Extremo Sul 
Catarinense, Departamento De Nutrição. Disponível Em: Https://Www.Scielo.Br/J/Tes/A/Pvdqhjvjnkszvx8r7v3cgnr/?Lang=Pt

Wopereis, Andresa. Avaliação Da Assistência Farmacêutica De Um Município Catarinense. 74f. Universidade Federal De Santa Catarina Curso De Graduação Em Farmácia Disciplina De Trabalho De Conclusão De Curso Ii. 2015. Disponível Em: Https://Repositorio.Ufsc.Br/Bitstream/Handle/123456789/159953/Tcc\%2oandressa\%2obo rgert\%20wopereis. Pdf?Sequence $=\mathrm{I} \&$ Isallowed $=\mathrm{Y}$

Barbosa, Marta. A Relação Da Automedicação Com A Farmácia Domiciliar: Uma Revisão De Literatura. 6of. Monografia Apresentada Ao Curso De Bacharelado Em Farmácia Da Faculdade Maria Milza, Como Requisito Para Obtenção Do Título De Graduado. 2017. Disponível Em: Http://Famamportal.Com.Br:8o82/Jspui/Bitstream/123456789/580/I/Monografia\%20\%20 farm\%C3\%Aicia\%202017.2.Pdf

Marangon, Jaciara. Educação Em Saúde E Serviço Farmacêutico Em Uma Farmácia Comunitária: Relato De Experiência. i8f. Curso De Pós-Graduação Lato Sensu Em Gestão E Atenção Farmacêutica. 2018. Disponível Em: Https://Bibliodigital.Unijui.Edu.Br:8443/Xmlui/Bitstream/Handle/123456789/5555/Jacia ra\%20alegransi\%2omarangon.Pdf?Sequence $=\mathrm{I} \&$ Isallowed $=\mathrm{Y}$ 